\title{
ROTAXANOS - INTERAÇÕES INTERCOMPONENTES E MOVIMENTOS MOLECULARES
}

\author{
Fellipe Freire Santos Fariasa, $^{a}$, Tainára Orlando, ${ }^{a}$,, Paulo Roberto dos Santos Salbego ${ }^{a}$,(®) Marcos Antonio Pinto \\ Martins ${ }^{\mathrm{a}, *},(1)$ \\ aDepartamento de Química, Universidade Federal de Santa Maria, 97105-900 Santa Maria - RS, Brasil
}

Recebido em 27/05/2020; aceito em 15/07/2020; publicado na web em 31/08/2020

\begin{abstract}
ROTAXANES - INTERCOMPONENT INTERACTIONS AND MOLECULAR MOVEMENTS. Rotaxanes are currently one of the most promising objects of study in the field of artificial molecular machines. A rotaxane molecule is composed by two or more molecular components that are connected by a mechanical bond. 51 years ago, the first synthesis of this type of molecules was performed, since then, there has been a great effort in obtaining new models to study its molecular movements and application as molecular machines. Here, we present an overview of intercomponent interactions in rotaxanes and how these interactions affect the major movements presented by this class of molecules. For this, different approaches to assess these interactions are discussed, both in solution and solid state.
\end{abstract}

Keywords: rotaxanes; intercomponent interactions; molecular machines; molecular movements; supramolecular chemistry.

\section{INTRODUÇÃO}

A síntese de moléculas através da formação de ligações covalentes é a maneira mais utilizada para obter novos modelos. No entanto, desde a década de 60 , a formação de moléculas consistindo em componentes conectados entre si, sem estarem ligados covalentemente, é relatada. ${ }^{1-3}$ Esses compostos formam a classe de moléculas mecanicamente entrelaçadas, do inglês, Mechanically Interlocked Molecules (MIMs). ${ }^{3}$

Métodos de síntese desta nova classe de compostos foi uma descoberta de grande relevância, já que esses compostos não são encontrados na natureza, tornando assim a química, e a própria ideia de existência dessas moléculas, ainda mais inovadora. Embora na época, a dimensão da sua utilidade não poderia ser imaginada, hoje sabe-se da sua variedade de aplicações. ${ }^{4-9}$

As MIMs possuem a característica intrínseca da ligação mecânica, ${ }^{10-14}$ ou seja, os componentes destas moléculas estão unidos por consequência de suas topologias, sendo que para ocorrer a dissociação é necessário o rompimento ou distorção significativa de ligações covalentes dos componentes. ${ }^{11}$ Nesse contexto, é encontrado um tipo de MIM: o rotaxano.

Os rotaxanos são constituídos basicamente de um componente linear com grupos volumosos nas extremidades (conhecido como eixo molecular) e um macrociclo, podendo conter mais de uma unidade de cada componente. ${ }^{15-18}$ Os grupos volumosos nas extremidades possuem a função de impedir a saída do macrociclo ${ }^{19-21}$ e o local onde está localizado o macrociclo no eixo molecular é conhecido como estação (Figura 1). ${ }^{22}$

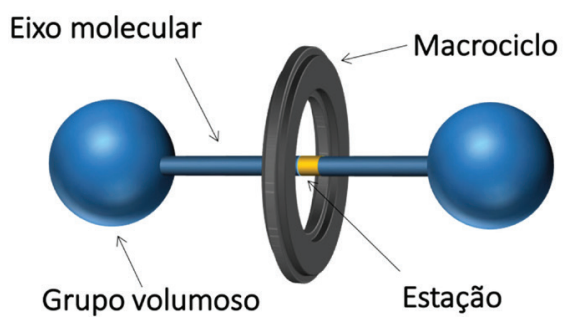

Figura 1. Representação genérica de uma molécula de [2]rotaxano

\footnotetext{
*e-mail: marcos.nuquimhe@gmail.com
}

Etimologicamente, a palavra rotaxano deriva de palavras do latim e possuem relação direta com sua estrutura molecular - onde rota significa roda e axis denota o eixo. ${ }^{23-25} \mathrm{Na}$ nomenclatura dos rotaxanos (de acordo com as recomendações da IUPAC (2012)), ${ }^{26}$ o prefixo entre colchetes " $[\mathrm{X}]$ " indica o número de componentes interconectados na molécula. ${ }^{26}$ Nesse sentido, na Figura 1 está apresentado um [2] rotaxano, em que há um eixo molecular e um macrociclo, resultando em um total de 2 componentes.

Alguns rotaxanos podem desencadear movimentos mecânicos controlados através de estímulos químicos ou físicos, mimetizando assim maquinários do cotidiano, dessa forma, sendo classificadas como máquinas moleculares. ${ }^{27,28} \mathrm{~A}$ maior parte das máquinas e motores moleculares artificiais projetados ultimamente é baseada em rotaxanos. ${ }^{29-31}$ Assim, os tipos de interações intercomponentes presentes, ou seja, entre o eixo molecular e o macrociclo, estão diretamente relacionadas a esses movimentos, ${ }^{18,32-35}$ afetando como eles são exercidos. ${ }^{36,37}$ Consequentemente, entender como os movimentos são afetados e como podem ser controlados e executados torna-se relevante.

Compreender as interações intercomponentes presentes nessas moléculas, em solução e no estado sólido, têm importância principalmente no planejamento e design de novos rotaxanos. Seja, permitindo um projeto sintético mais eficiente para novos modelos, ou ainda, possibilitando pré-estabelecer o controle e magnitude de movimentos, para o uso de rotaxanos como máquinas moleculares. ${ }^{34,38}$ Para a aquisição de informações sobre as interações, um conjunto de ferramentas e experimentos podem ser utilizados, tais como espectroscopia de Ressonância Magnética Nuclear (RMN), 32,33,39,40 espectroscopia no infravermelho, ${ }^{41,42}$ espectroscopia no ultravioleta ${ }^{43}$ e cálculos de mecânica quântica, ${ }^{44,45}$ os quais podem ser aliados a dados obtidos por experimentos de difração de raios $\mathrm{X}$ de monocristal (SC-XRD). ${ }^{18,46}$

Os estudos relacionados às moléculas de rotaxanos vêm crescendo desde a virada do milênio, envolvendo tópicos que permeiam desde a síntese dessas estruturas até suas aplicações. ${ }^{4-9}$ A importância do tema foi destacado em 2016, quando a Academia Real Sueca de Ciências concedeu o prêmio Nobel de Química a três cientistas: Jean-Pierre Sauvage, James F. Stoddart e Bernard L. Feringa, pelo "design e síntese de máquinas moleculares". ${ }^{10,13}$

A dimensão desse campo de pesquisa está refletida no número de trabalhos publicados e no índice h durante o período do início dos anos 
2000 a junho de 2020 (Figura 2). O termo "rotaxane *" foi usado como parâmetro de pesquisa no banco de dados Web of Science (Principal Coleção), resultando em um total de 3562 publicações e um índice $h$ de 159. O Brasil apresenta um singelo número de publicações na área, mas sua contribuição vem crescendo, como é possível perceber nos números dos últimos 5 anos (Figura 2).

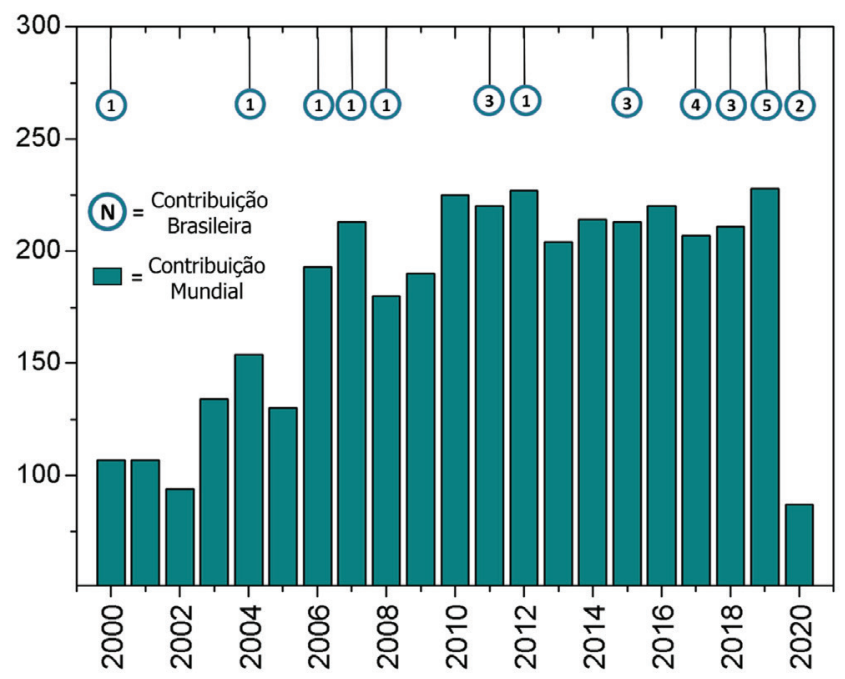

Figura 2. Número de artigos publicados durante janeiro de 2000 - junho de 2020 incluindo o termo "rotaxane *"; na parte superior do gráfico, os círculos indicam a contribuição brasileira em cada ano

Neste contexto, o objetivo desta revisão é apresentar de maneira concisa um panorama das principais interações intercomponentes presentes em moléculas de [2]rotaxanos. O presente review não tem o intuito de pontuar todos os artigos que tratam sobre interações intercomponentes. Mas sim, busca destacar os principais conceitos envolvidos na construção do conhecimento sobre essas interações nos últimos 50 anos. Serão descritos os principais tipos de interações, como avaliá-las em solução e no estado sólido, destacando a contribuição da pesquisa brasileira na área, e a correlação entre as interações e os movimentos executados pelos rotaxanos.

\section{INTERAÇÕES INTERCOMPONENTES E MOVIMENTOS}

Por decorrência da proximidade espacial dos componentes moleculares, diferentes interações intercomponentes poderão existir. $^{24,47}$ As interações emergem como consequência do entrelaçamento entre os componentes e estão diretamente relacionadas com os movimentos executados por essas moléculas. ${ }^{18,32-35}$ Isso porque, para que um componente se movimente em relação ao outro, é necessário que interações intercomponentes sejam rompidas e, consequentemente, novas interações sejam formadas.

\section{Tipos de interações presentes em [2]rotaxanos}

Vamos iniciar recordando como surgiu o interesse em modelar as interações nessas moléculas e, para isso, precisamos relembrar um pouco do surgimento das moléculas de rotaxano.

O termo rotaxano foi introduzido por Gottfried Schill e Hubertus Zollenkopf no ano de 1967. ${ }^{25}$ Logo após, em 1969, eles demonstraram a síntese de uma molécula de [2]rotaxano em que os componentes estavam previamente interligados por ligações covalentes, e que, subsequentemente essas ligações eram quebradas, deixando somente a ligação mecânica entre os componentes. ${ }^{25}$

Ao mesmo tempo, em 1967, Harrison \& Harrison por meio do método de uma abordagem estatística demonstraram a formação de rotaxanos ${ }^{48} \mathrm{em}$ que a organização das moléculas ocorria de maneira aleatória, sem qualquer força atrativa entre as espécies moleculares. Esse experimento foi repetido cerca de 70 vezes pelos autores, resultando em uma média de $6 \%$ de rendimento. ${ }^{48,49}$ Desde então, os rotaxanos vêm sendo obtidos com maior praticidade e versatilidade, sendo o planejamento e uso das interações que ocorrem entre os componentes, uma das causas desse avanço no planejamento sintético. ${ }^{17,24}$ Assim, atualmente é comum observar interações sendo utilizadas como template na síntese e no entrelaçamento dessas estruturas.

É importante ressaltar que, as interações intercomponentes presentes nas moléculas de rotaxano, não são apenas aquelas que foram (ou serão) utilizadas como template para a síntese, mas sim, todas as possíveis interações que ocorrem entre os componentes de uma molécula de rotaxano durante e depois da síntese, até mesmo as interações ditas como fracas ou dispersivas. ${ }^{33,34}$ Além das interações entre a estação do eixo molecular e o macrociclo, podem ser verificadas interações com os grupos volumosos nas extremidades e outras porções do eixo molecular, acarretando em uma maior estabilidade da molécula e interferindo diretamente nos movimentos exercidos. ${ }^{34}$ Nesse sentido, a participação de todas essas interações (Figura 3) na estabilização da estrutura devem ser consideradas quando é buscado compreender o movimento de tais moléculas.

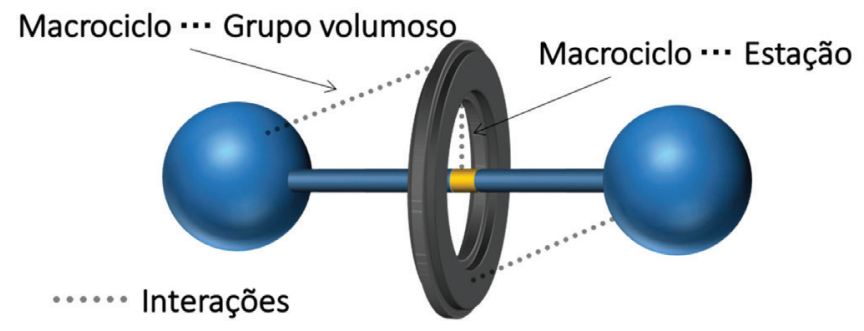

Figura 3. Representação das interações possíveis entre eixo molecular e macrociclo

Entre as principais interações intercomponentes presentes em rotaxanos, podemos citar as ligações de hidrogênio, ${ }^{15,34,38}$ coordenação de íons metálicos, ${ }^{50-53}$ empilhamento $\pi \cdots \pi,{ }^{54-57}$ interações de íons, ${ }^{58-60}$ interações hidrofóbicas ${ }^{61}$ e de halogênio. ${ }^{62}$ Algumas dessas interações intercomponentes podem ser observadas na Figura 4.

Dependendo da natureza da interação formada entre os componentes dos rotaxanos, pode haver a presença de um ou múltiplos sítios de interação entre os componentes. Como já mencionado, alguns destes sítios podem estabilizar e auxiliar na formação da ligação mecânica. ${ }^{63,64} \mathrm{Um}$ dos exemplos são os rotaxanos do tipo Leigh (Figura 4 (a)), em que o macrociclo possui grupos doadores de ligação de hidrogênio $(\mathrm{NH})$ e eixos moleculares que possuem átomos aceptores de ligações de hidrogênio (e.g., grupos carbonila). ${ }^{34,38,46}$ Assim, ligações de hidrogênio, como interações $\mathrm{N}-\mathrm{H} \cdots \mathrm{O}$ e $\mathrm{C}-\mathrm{H} \cdots \mathrm{O}$, serão formadas direcionando a formação da molécula entrelaçada, porém, as ligações de hidrogênio não serão as únicas a existirem após a formação do rotaxano, existirão também diversas outras interações entre o eixo molecular e o macrociclo. ${ }^{34}$ Sendo assim, o somatório dessas interações é cooperativo, auxiliando na estabilização da estrutura do rotaxano. ${ }^{23,34}$

\section{Movimentos realizados por rotaxanos}

O movimento de um rotaxano é dado pela característica intrínseca da ligação mecânica. Essas moléculas têm a capacidade de sofrer movimentos controlados de um componente em relação ao outro. Na literatura, são reportados movimentos como: rotacional (pirouetting), ${ }^{24,32}$ translacional (shuttling), ${ }^{24,55}$ pendular (rocking) ${ }^{19,65} \mathrm{e}$ 


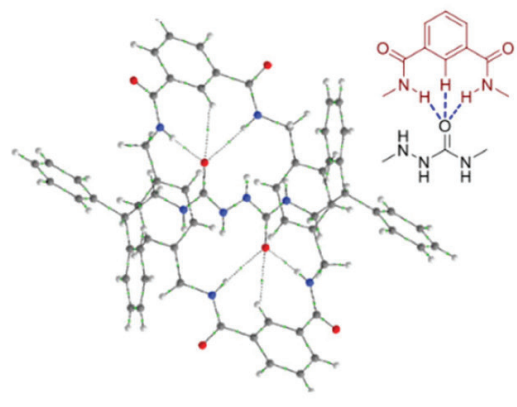

(a)

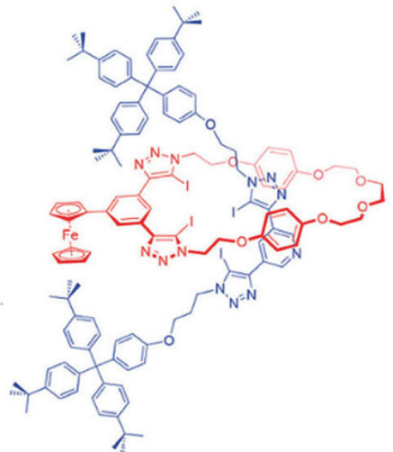

(b)

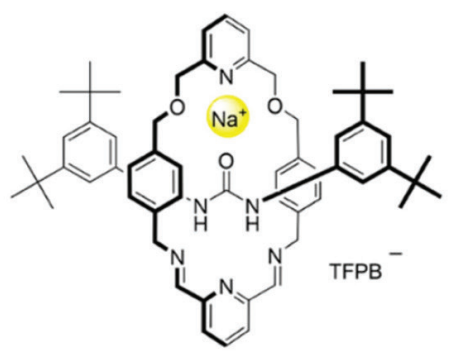

(c)

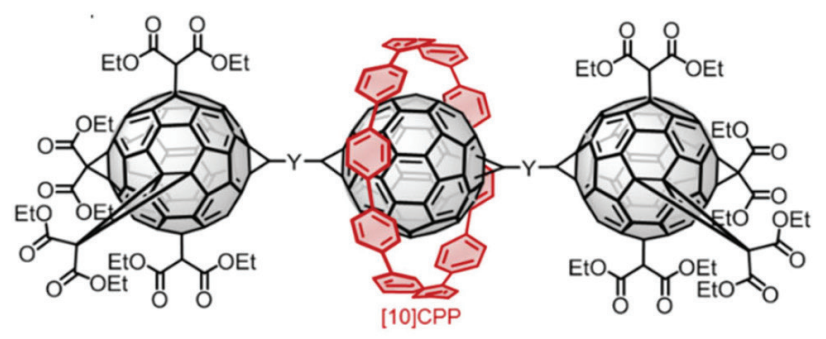

(d)

Figura 4. Representação das interações presentes entre os componentes dos rotaxanos mais recorrentes na literatura. (a) Ligações de hidrogênio [reproduzido e adaptado com a permissão de Salbego \& Martins et al., Eur. J. Org. Chem. 2019, 3464-3471. Copyright (2019) Wiley Online Library]; (b) modelo com metal-ativo [reproduzido com a permissão de Lim \& Beer, Eur. J. Org. Chem. 2019, 3433-3441. Copyright (2019) Wiley Online Library]; (c) modelo contendo íon [reproduzido e adaptado com a permissão de Chiu, Chem. Eur. J. 2014, 20, 4563-4567. Copyright (2014) Wiley Online Library]; (d) empilhamento $\pi \cdots \pi$ [reproduzido com a permissão de Guldi \& Delius et al., J. Am. Chem. Soc. 2018, 140, 41, 13413-13420. Copyright (2018) American Chemical Society]

a respiração do macrociclo (breathing), ${ }^{40}$ representados na Figura 5. Assim, os rotaxanos são protótipos apropriados para a construção de múltiplos dispositivos moleculares, rotativos e/ou de deslocamento. ${ }^{24,28}$

Dentre os diversos movimentos efetuados pelos rotaxanos, na literatura são estudados principalmente dois movimentos. Onde o macrociclo se locomove translacionalmente e gira em relação ao eixo molecular, ou seja, o movimento de translação e rotação, respectivamente. ${ }^{18,32,66-69}$
No movimento rotacional (Figura 5 (a)), o macrociclo possui uma rotação relativa ao eixo molecular. Há várias possibilidades de interferir na taxa de rotação de um macrociclo, como: variando a temperatura, ${ }^{18,70}$ variando a intensidade de campo elétrico,${ }^{71} \mathrm{com}$ o uso de luz mudando assim o comprimento de onda, ${ }^{72}$ ou usando a própria liberdade estrutural da molécula, alterando o meio em que está inserido (e.g., solvente). ${ }^{73} \mathrm{~A}$ frequência de rotação de um macrociclo ao redor de um eixo molecular depende fortemente das interações

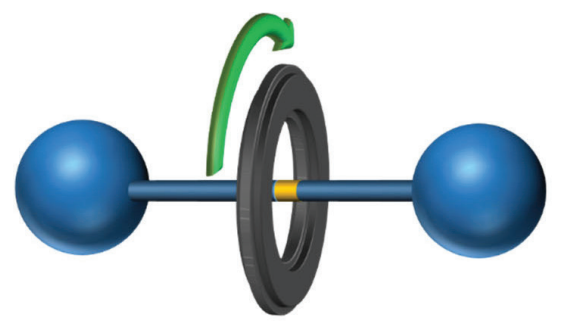

(a) Rotação

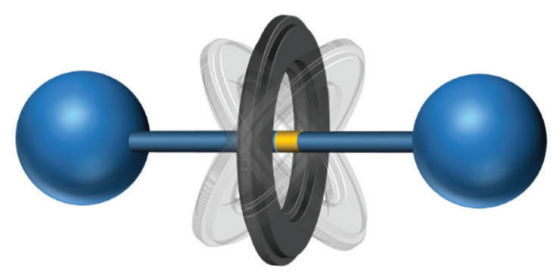

(c) Pendular

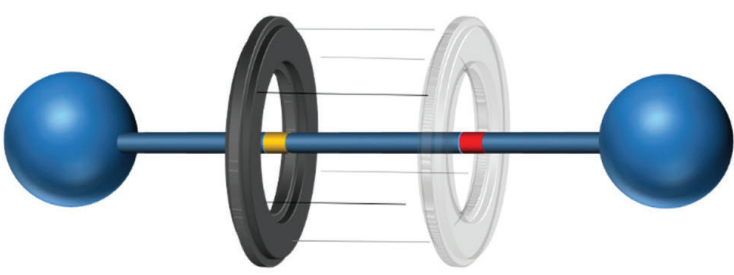

(b) Translação

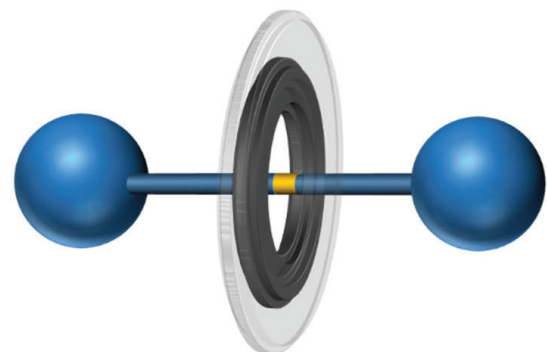

(d) Respiração

Figura 5. Representação de alguns dos movimentos que podem ser executados por moléculas de [2]rotaxanos 
intercomponentes e de interações com o meio que a molécula está presente. ${ }^{73}$

Já no movimento de translação (Figura 5 (b)) a molécula deve possuir mais de um sítio de interação entre os componentes, i.e., mais de uma estação, ${ }^{22}$ como demonstrado na Figura 5 (b) (estações representadas nas cores amarelo e vermelho do eixo molecular). Stoddart e colaboradores reportaram a primeira molécula com duas estações em 1991 e desde então, outros exemplos surgiram usando associações similares. ${ }^{22,73}$

O movimento de translação apresenta diversos fatores que podem interferir no comportamento das moléculas, assim o controle desse movimento é desafiador como também promissor. ${ }^{24,74}$ Dentre esses fatores podemos citar: o comprimento do eixo molecular, ${ }^{75,76} \mathrm{o}$ número de estações, ${ }^{76,77}$ a distância entre as estações, ${ }^{78}$ a rapidez de deslocamento, ${ }^{78}$ a natureza das interações, entre outros. ${ }^{75,78}$

Outros movimentos mostrados na Figura 5 são menos explorados, pois são movimentos oriundos da junção dos movimentos de rotação e translação. ${ }^{40}$ No caso do movimento pendular (Figura 5 (c)), o macrociclo possui uma unidade dipolar passível de um movimento de oscilação. Quando aplicado um campo elétrico externo ao sistema, por exemplo, o movimento pendular pode ser desacelerado ou interrompido devido a interação do dipolo com o campo externo. ${ }^{65}$

Há a possibilidade também da respiração do macrociclo, em que movimentos correlatos à contração e/ou expansão do macrociclo são partes desse processo mais global. Nesse movimento, ocorre uma mudança estocástica da cavidade do macrociclo devido a vibração das ligações ou rotação diédrica, que leva a modificação nas interações intercomponentes e possibilita outros processos dinâmicos. ${ }^{40}$

Fazendo um paralelo com o nível macroscópico, as máquinas que utilizamos dependem do movimento controlado de múltiplos componentes para realizar tarefas específicas. ${ }^{10,73}$ Dessa forma, buscando chegar em uma situação na qual as máquinas moleculares sintéticas sejam úteis no nosso cotidiano tanto quanto as máquinas macroscópicas, os esforços estão concentrados na direção de estabelecer métodos para controlar o posicionamento e o movimento dos componentes moleculares. ${ }^{79,80}$

Uma vez que as máquinas operam em ciclos repetidos, um requisito importante para as máquinas moleculares é que as mudanças ocorridas ou reações químicas realizadas no sistema sejam reversíveis. ${ }^{73}$ Sendo assim, alguns processos químicos ou físicos que atendem a esse requisito, foram reportados, como: reações ácido/base, ${ }^{79,73}$ isomerizações, ${ }^{19,81}$ métodos de oxidação/ redução, ${ }^{45,82}$ equilíbrios de complexação/descomplexação, ${ }^{62,83}$ reações que envolvem a formação e quebra de interações de hidrogênio, ${ }^{18,51,81}$ reações fotoquímicas, ${ }^{19,84}$ eletroquímicas ${ }^{84-86}$ e termoquímicas. ${ }^{10,28}$

\section{Interações intercomponentes em solução}

Experimentos de RMN em solução ${ }^{32,66}$ podem ser empregados para estudar os movimentos realizados por rotaxanos. Isso porque átomos que possuem diferentes ambientes químicos terão diferentes deslocamentos químicos. Como o ambiente químico de um núcleo observado é modificado simultâneo ao movimento ocorrido, movimentos de rotação e translação em moléculas de rotaxanos podem ser monitorados. ${ }^{87}$ Isso fornecerá informações para elucidar como esses movimentos ocorrem, como podem ser modificados e controlados.

Rotaxanos que, por exemplo, possuem macrociclos de tetralactama, geralmente adotam a co-conformação cadeira, similar a um ciclohexano. ${ }^{71,88}$ Nessa co-conformação pode ser verificada a presença de hidrogênios em posições pseudoaxiais e pseudoequatoriais (Figura 6, hidrogênios E e E'). Com isso, é possível aferir o movimento rotacional utilizando experimentos de RMN de ${ }^{1} \mathrm{H}$ em solução. É possível observar esse fenômeno em uma das moléculas sintetizadas e caracterizada por nosso grupo de pesquisa ${ }^{89}$ (Figura 6).

Ao ser verificado apenas um sinal (Figura 6, $313 \mathrm{~K}$, temperatura de coalescência) para esses dois hidrogênios (E e E') é indicativo de um movimento dinâmico rotacional rápido na escala de tempo do RMN. Entretanto, quando resfriada a amostra, pode ser constatada a resolução de dois sinais diferentes, ou seja, é possível verificar os hidrogênios em dois ambientes magneticamente distintos, o que indica que está ocorrendo um movimento mais lento na escala temporal do RMN (Figura 6, 298 K). Assim, é possível acompanhar o movimento de rotação de um rotaxano. ${ }^{23,87,90-92}$

Nesse sentido, o RMN se tornou o método mais aplicado na observação e monitoramento de movimentos intercomponentes com mudança de conformação, na qual a energia livre de ativação, na

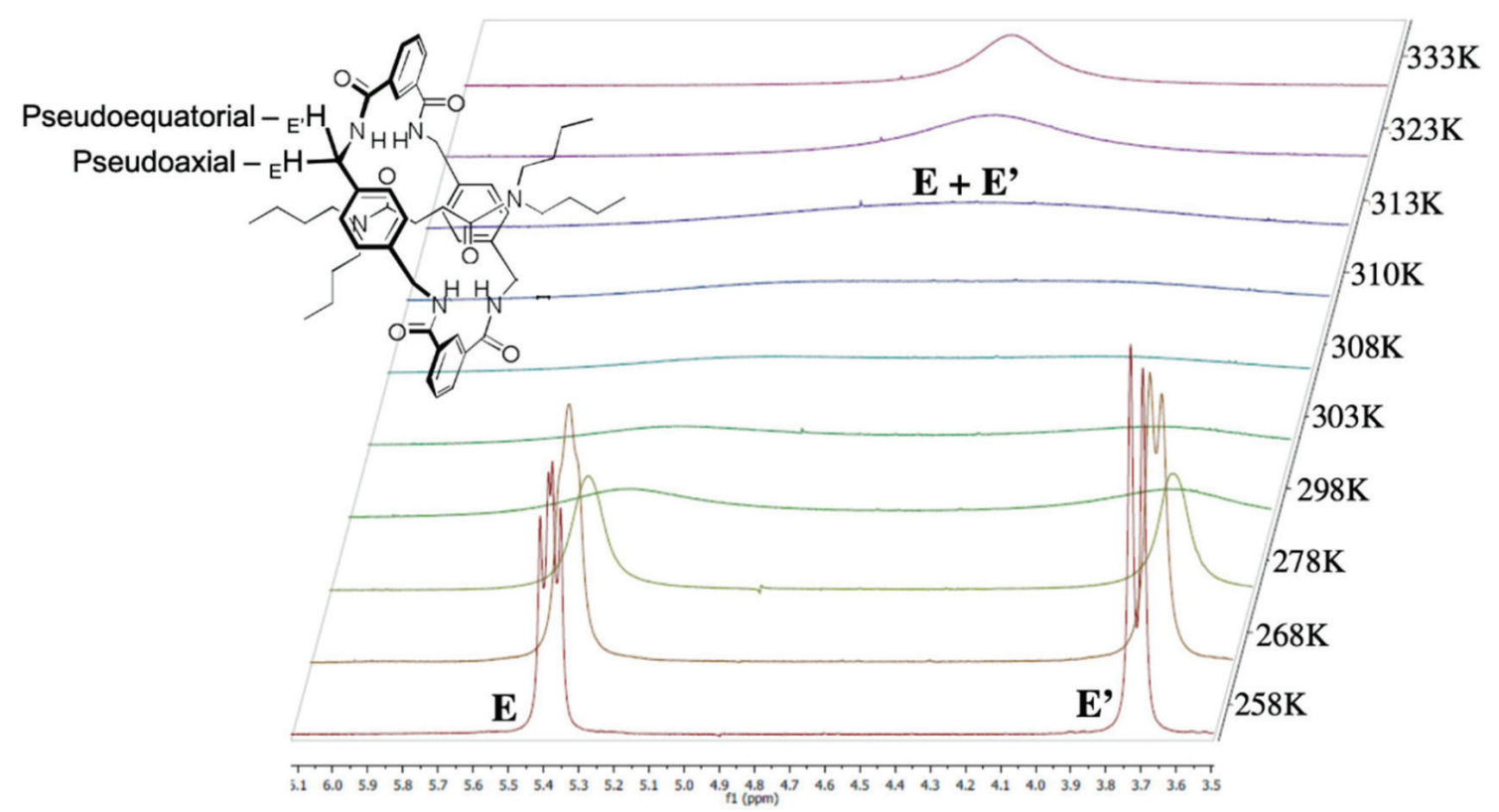

Figura 6. Representação da co-conformação cadeira adotada por um [2]rotaxano contendo um macrociclo de tetralactama e o fenômeno de coalescência para os hidrogênios E e E', pseudoaxial e pseudoequatorial, respectivamente. Os espectros de RMN de ${ }^{1} H$ foram obtidos em diferentes temperaturas e estão expandidos na faixa de 3.5 a $6.1 \mathrm{ppm}\left(400 \mathrm{MHz}, \mathrm{CDCl}_{3}\right.$ ) [reproduzido e adaptado de Rodrigues, L. V. ${ }^{89} \mathrm{CC}$ BY-NC-ND 4.0 License (2013)] 
temperatura de coalescência, comumente é encontrada na faixa entre 6 à $25 \mathrm{kcal} \mathrm{mol}^{-1}$, para [2] rotaxanos com macrociclos de tetralactama. ${ }^{91}$

A mudança estrutural no eixo molecular da molécula de rotaxano interfere diretamente na energia livre de ativação ${ }^{18,20,33}$ e a natureza das interações entre os componentes reflete nos movimentos. ${ }^{66}$ Estudos que utilizaram uma variação dos grupos volumosos ${ }^{20,32}$ ou no diâmetro do macrociclo, ${ }^{20,93}$ demonstraram que as interações entre o macrociclo e os grupos volumosos mudam significativamente a energia de ativação dos movimentos. Nosso grupo de pesquisa demonstrou que inclusive confôrmeros de [2] rotaxanos são influenciados pela mudança estrutural mudando as interações entre os componentes e consequentemente a energia de ativação do movimento. ${ }^{33}$ Adicionalmente, experimentos com variação de temperatura também são realizados para análise dos tipos de interações envolvidas. ${ }^{18,33}$

Analisando espectros de uma molécula de [2] rotaxano contendo macrociclo de tetralactama (Figura 7), percebemos mudanças no deslocamento químico de certos hidrogênios $\left(C, D, a, e_{8 A}{ }_{8 \mathrm{~B}}\right.$ e e' Figura 7), com experimento de variação da temperatura. As principais interações percebidas em solução são as ligações de hidrogênio entre o macrociclo e a estação, e interações $\mathrm{C}-\mathrm{H} \cdots \pi$ entre o macrociclo e o grupo volumoso.

A desblindagem dos hidrogênios C e D é característico de quando o átomo de hidrogênio está participando de uma ligação de hidrogênio (e.g., $\mathrm{NH} \cdots \mathrm{O}$ ), isso porque os átomos mais eletronegativos estão atraindo a densidade eletrônica para si, deixando o hidrogênio mais suscetível a sentir o efeito do campo magnético exercido pelo $\mathrm{RMN}^{94}$ (Figura 7). Quando a interação é rompida, com o aumento da temperatura, o hidrogênio volta a ser blindado e, no espectro de $\mathrm{RMN}$, é movido para deslocamentos químicos menores. ${ }^{71} \mathrm{~A}$ formação de uma interação $\mathrm{C}-\mathrm{H} \cdots \pi$ é caracterizada pela blindagem do sinal do hidrogênio que participa desta interação. Isso ocorre devido ao efeito anisotrópico exercido pelos anéis benzílicos presentes no macrociclo, blindando os hidrogênios quando estão espacialmente dentro do cone de blindagem anisotrópico. ${ }^{95} \mathrm{O}$ rompimento da interação $\mathrm{C}-\mathrm{H} \cdots \pi$ com o aumento de temperatura é caracterizado pela desblindagem dos hidrogênios e' ${ }_{8 \mathrm{~A}}$ e e' ${ }_{8 \mathrm{~B}}$, como observado na Figura 7.

Além do movimento de rotação, experimentos de RMN podem ser utilizados para observar outros movimentos característicos, ${ }^{96}$ como o de translação, ou seja, a capacidade de locomoção do macrociclo ao longo do eixo molecular.

Esse movimento, em particular, foi primeiramente observado por L. Anelli, N. Spencer e J. F. Stoddart em 1991, ${ }^{97}$ ao utilizarem uma molécula de [2]rotaxano com um eixo molecular contendo duas estações. Os autores puderam rastrear em qual das estações permanecia o macrociclo, por meio de experimentos de RMN de ${ }^{1} \mathrm{H}$ com variação de temperatura. ${ }^{97}$ Nesse trabalho, os autores declararam, em tradução livre: "Agora existe a oportunidade de explorar o movimento molecular [translacional] inserindo estações diferentes ao longo do eixo molecular [...] de tal maneira que essas diferentes estações possam ser alcançadas seletivamente por meios químicos, eletroquímicos ou fotoquímicos [...]. Na medida em que for possível controlar o movimento de um componente molecular em relação ao outro em um [2]rotaxano, a tecnologia para a construção de "máquinas moleculares" emergirá." fato, os primeiros exemplos de rotaxanos que contém mais de uma estação, que respondem a estímulos externos, foram descritos nos anos seguintes. ${ }^{98-100}$

Para que um rotaxano funcione no transporte molecular é necessário mais de uma estação no eixo molecular, sendo uma de maior afinidade energética (maior estabilidade), em que o macrociclo tende a ficar posicionado e outra de menor afinidade (menor estabilidade). ${ }^{23,96,101,102}$ Por meio de algum estímulo externo
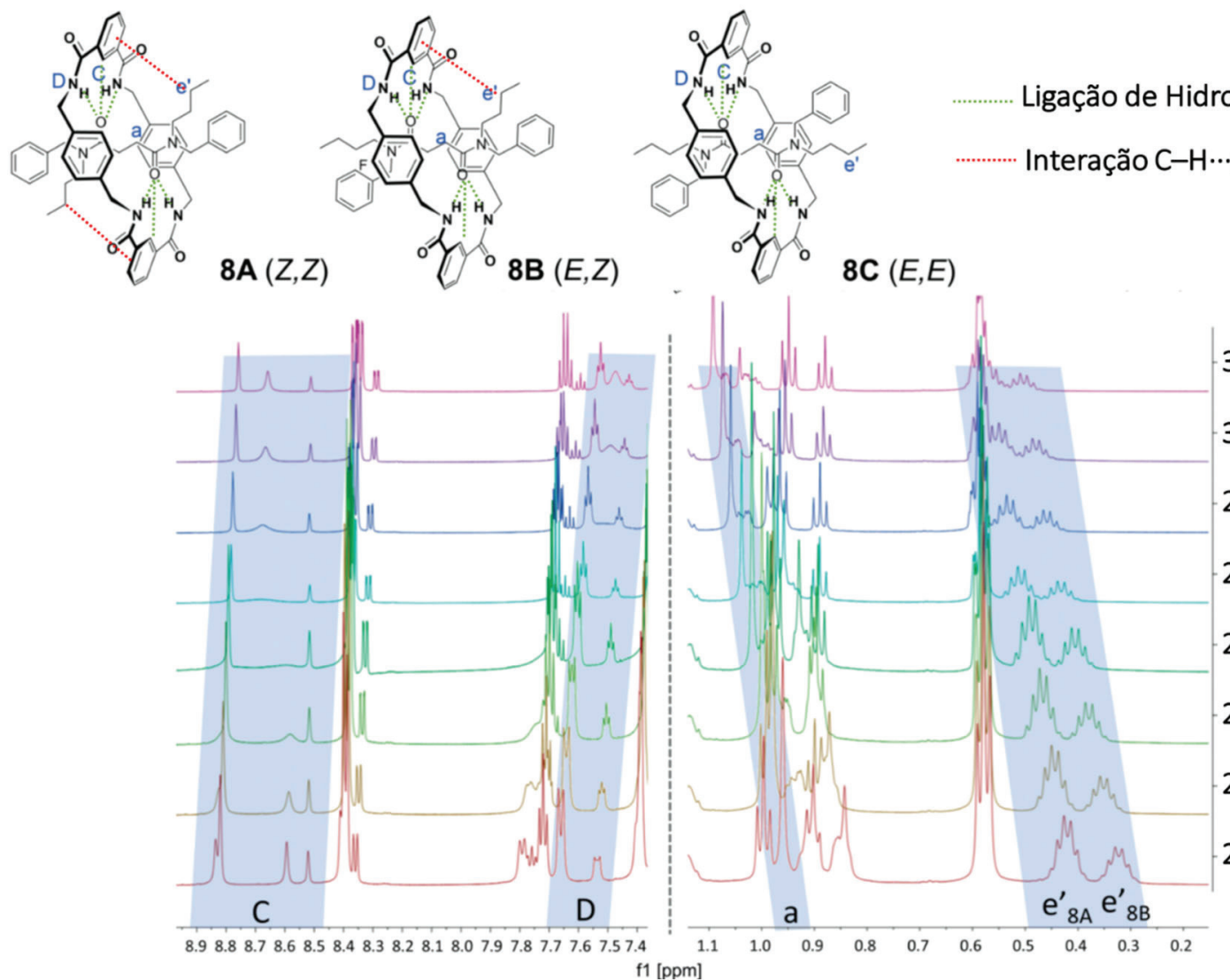

$313 \mathrm{~K}$

$303 \mathrm{~K}$

$293 \mathrm{~K}$

$283 \mathrm{~K}$

$273 \mathrm{~K}$

$263 \mathrm{~K}$

$253 \mathrm{~K}$

$243 \mathrm{~K}$

Figura 7. Espectros de RMN de ${ }^{1} \mathrm{H}$ em diferentes temperaturas, de confôrmeros de um [2]rotaxano contendo macrociclo de tetralactama, reportado por Orlando et al. (2018) [reproduzido e adaptado com a permissão de Martins et al., Eur. J. Org. Chem. 2018, 4978-4990. Copyright (2018) Wiley Online Library] 
(Estímulo 1, Figura 8), o macrociclo pode mudar de estação e se mover ao longo do eixo molecular, permanecendo posicionado no estado de menor afinidade, mas ainda na menor energia potencial depois do estímulo. $\mathrm{O}$ sistema pode ser restaurado ao estado original por meio de outro estímulo (Estímulo 2, Figura 8), e se esse processo puder ser controlado e sistematizado, teremos assim uma máquina molecular. ${ }^{103}$

A partir dos experimentos de RMN citados é possível obter dados para auxiliar na compreensão de como os movimentos podem ser controlados e como eles são originados. Tais experimentos, fornecem uma medida das energias de estabilidade das interações intercomponentes em cada estado, com o cálculo das barreiras energéticas. ${ }^{32,40}$

Interações intercomponentes também podem ser notadas observando-se o padrão espectroscópico dos componentes isolados vs. a molécula de rotaxano. Megiatto et al. (2018) publicaram uma síntese inovadora e altamente eficiente de rotaxanos a partir do método active-metal-template, baseada em porfirinatos de Co(II) como centro ativo. ${ }^{104}$ Como consequência dos grupos químicos presentes na estrutura final do rotaxano, uma ligação coordenada entre o grupo carbonila no eixo molecular e o íon Co(II) no macrociclo foi demonstrada. Esse tipo de interação pode ser confirmada em solução por experimentos de $\mathrm{RMN}$ de ${ }^{13} \mathrm{C}$ e no estado sólido por espectroscopia de infravermelho (FTIR-ATR). No caso do RMN de ${ }^{13} \mathrm{C}$, são comparados os espectros do eixo molecular, do macrociclo e do rotaxano. ${ }^{43,104}$

Apesar de uma boa parcela das informações fundamentais sobre os [2] rotaxanos ser proveniente de estudos em solução, também é importante a utilização de outros métodos para uma compreensão ampla desses sistemas. ${ }^{36}$

\section{Interações intercomponentes no estado sólido}

Correlacionando aos estudos em solução, as interações presentes nas moléculas de rotaxanos também podem ser analisadas no estado sólido. ${ }^{24,34,46}$ Há diversas maneiras de caracterizar interações no estado sólido, cada uma com diferentes particularidades, uma das mais recorrentes é a utilização de dados de difração de raios $\mathrm{X}$ de monocristal (SC-XRD, do inglês, Single Crystal X-Ray Diffraction). A SC-XRD é uma técnica analítica não destrutiva que fornece

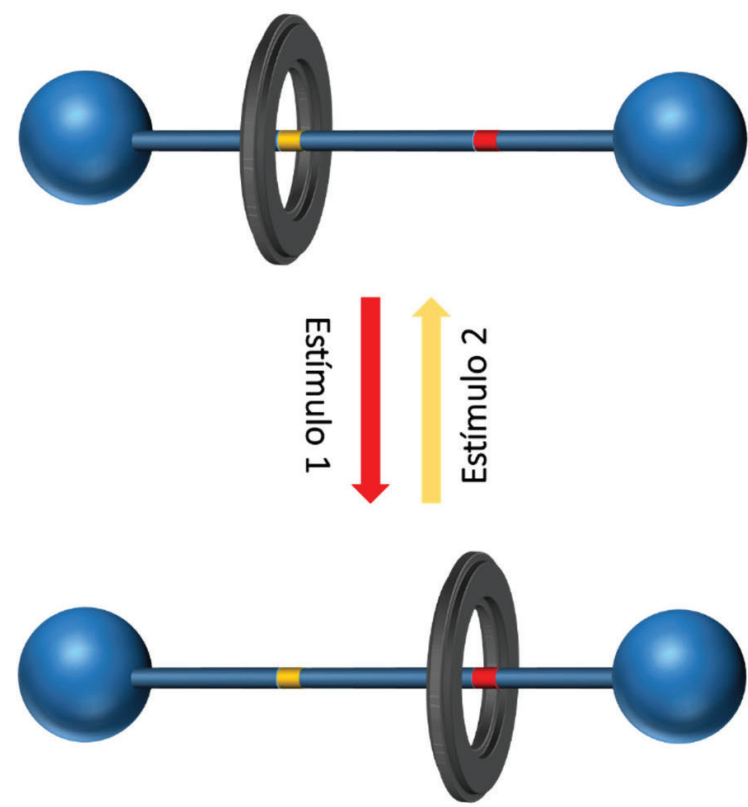

informações detalhadas sobre a estrutura de substâncias cristalinas, incluindo as dimensões unitárias das celas, comprimentos e ângulos de ligações e detalhes sobre o empacotamento cristalino. ${ }^{105}$

Com a adequada cristalização da molécula de rotaxano, é possível caracterizar o composto e identificar a sua estrutura, já que os dados provenientes de SC-XRD refletem de maneira íntegra como a molécula se encontra no estado sólido. Através da estrutura é possível constatar as interações intercomponentes e intramoleculares. Diversos estudos utilizam ângulos e distâncias entre átomos e/ou componentes para caracterizar interações, ${ }^{83,106,107}$ além disso, outros dados e características das moléculas também são obtidos, como: diâmetro do macrociclo, tamanho do eixo molecular e dos grupos volumosos. ${ }^{38,46}$

Uma investigação mais detalhada é imprescindível, uma vez que a formação e estabilidade de moléculas de rotaxanos dependem dos tipos de interações que existem entre os componentes. ${ }^{24}$ É observada uma tendência na literatura de classificar as interações utilizando uma abordagem geométrica, utilizando distâncias e ângulos (entre átomos e/ou planos, por exemplo), entretanto, abordando tal metodologia, o pesquisador pode negligenciar certas interações ao escolher quais interações ele considera adequadas. ${ }^{34}$

O nosso grupo de pesquisa tem levado em consideração além dos dados geométricos, as áreas de contato e a energia de estabilização entre os componentes, para determinar a contribuição das interações envolvidas na estabilização dessas moléculas. ${ }^{46}$ A partir de dados obtidos pela SC-XRD, podemos observar que os componentes adotam a melhor conformação com o propósito de maximizar as interações intercomponentes, ${ }^{46}$ além de buscar a maior eficiência do empacotamento cristalino, em que entram fatores topológicos, buscando a melhor organização molecular individual em relação ao todo, ou seja, a rede cristalina. ${ }^{108}$

Cálculos de mecânica quântica e estudos teóricos computacionais são muitas vezes empregados para verificar as energias envolvidas na estabilização das moléculas e nos movimentos moleculares. Baseados principalmente na Teoria do Funcional de Densidade (de sigla em inglês, DFT - Density Functional Theory) ${ }^{109}$ os estudos teóricos cobrem uma vasta gama, que incluem a dinâmica, modelagem e simulações moleculares. ${ }^{34,39,46,71,110-113}$ As energias de estabilização entre os componentes do rotaxano, podem ser obtidas utilizando estruturas cristalinas medidas por SC-XRD através de cálculos DFT, utilizando a Equação 1 . Na equação $1, G_{M c y \cdot T h r}$ refere-se à energia de

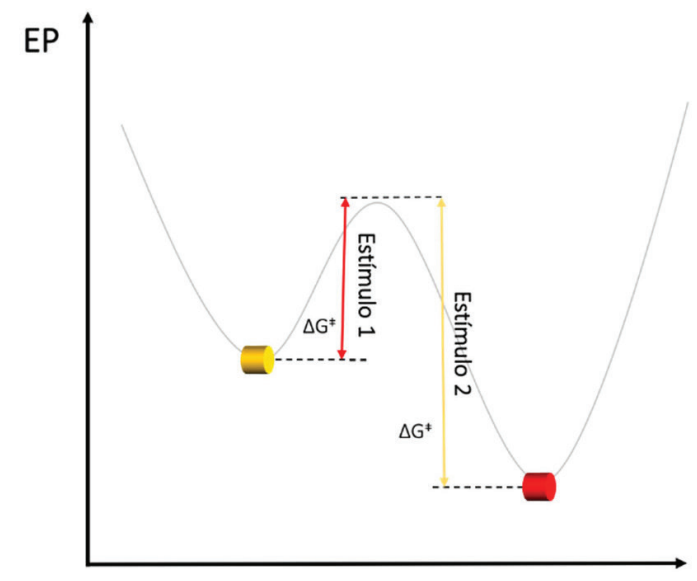

Figura 8. Representação energética da dinâmica do movimento de translação em relação a estímulos externos 
estabilização entre o macrociclo (Mcy) e eixo molecular (Thr), $G_{R o t}$ denota a energia de estabilização obtida para a molécula de rotaxano, e $G_{M c y}$ e $G_{T h r}$ refere-se às energias de estabilização dos componentes separados. $^{34}$

$$
G_{M c y \cdots T h r}=G_{R o t}-\left(G_{M c y}+G_{T h r}\right)
$$

Além disso, a identificação de cada interação intercomponente pode ser obtida utilizando a Teoria Quântica dos Átomos nas Moléculas, do inglês, Quantum Theory of Atoms In Molecules (QTAIM). ${ }^{114,115}$ A QTAIM é uma teoria bastante útil para estudar a estrutura eletrônicas das moléculas, e dentre suas diversas aplicabilidades, ela é empregada para estudar caminhos de interações entre átomos. ${ }^{116}$ Nos rotaxanos, a QTAIM pode indicar caminhos de interações entre os átomos dos componentes ou das moléculas. ${ }^{33-35,46}$ A QTAIM fornece uma descrição quântica do comportamento e localização dos átomos em uma ligação química, além disso, providencia a densidade de elétrons, conhecida como $\rho$, de cada ponto crítico da ligação (de sigla em inglês, BCP - Bond Critical Point), ou seja, o mínimo da densidade de elétrons no caminho da ligação (ou interação). ${ }^{15,116}$

Uma vez obtidos ambos os dados, energia de estabilização entre os componentes e $\rho$ de cada interação intercomponente, os autores utilizaram a análise $\mathrm{G}_{\mathrm{AI}}{ }^{117-121}$ para esse tipo de sistema. ${ }^{34} \mathrm{~A}$ análise $\mathrm{G}_{\mathrm{AI}}$ fornece a energia de interação átomo $\cdots$ átomo, distribuindo a energia de estabilização $G_{M c y \cdots T h r}$ entre cada caminho de interação, de acordo com a Equação 2. ${ }^{34}$ Em que $\rho$ é o valor de densidade eletrônica de cada interação e $\Sigma \rho$ é o somatório da densidade eletrônica de todos os caminhos de interação presentes entre os componentes do rotaxano

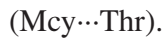

$$
G_{A I}=G_{M c y \cdots \mathrm{Thr}} \times\left(\frac{\rho}{\sum \rho}\right)
$$

Salbego e colaboradores ${ }^{34}$ investigaram a classificação e a quantificação adequada de ligações de hidrogênio intercomponentes presentes no estado sólido de [2]rotaxanos portadores do macrociclo de tetralactama, utilizando 14 estruturas cristalinas com diferentes eixos moleculares. ${ }^{34}$ A principal discussão se baseou em interpretações antecipadas sobre as ligações de hidrogênio principais nesses modelos, frequentemente classificada de ligação de hidrogênio bifurcada, por causa da presença de interações ditas "clássicas" $(\mathrm{N}-\mathrm{H} \cdots \mathrm{O})$ e informações sobre interações ditas "fracas" $(\mathrm{C}-\mathrm{H} \cdots \mathrm{O})$ eram geralmente omitidas ou negligenciadas. Entretanto, foi indicado que as ambas interações podem possuir energia de estabilização similar e até mesmo as ditas "fracas" podem ter energias de estabilização superiores às "clássicas". ${ }^{34}$

Nesse trabalho, os autores demonstraram outras abordagens utilizadas para avaliar as interações intercomponentes em uma molécula de [2]rotaxano, neste caso, especialmente o conjunto de ligações de hidrogênio entre o macrociclo e eixo molecular (Figura 9). A molécula utilizada como exemplo, foi relatada por Berná e colaboradores (2012), ${ }^{32}$ com ela como modelo, são demonstradas as distâncias e ângulos referentes a abordagem geométrica (Figura 9 (a)); os caminhos de interação entre os átomos gerados a partir do cálculo utilizando QTAIM (Figura 9 (b)); a área de contato (em $\AA^{2}$ ) entre os componentes, que é obtida através do Poliedro de VoronoiDirichlet, ${ }^{122}$ do inglês Voronoi-Dirichlet Polyhedra (VDP) usando o software ToposPro ${ }^{123}$ (Figura 9 (c)). Além de demonstrar a complementariedade entre as moléculas através da superfície de Potencial Eletrostático Molecular, ${ }^{124}$ do inglês Molecular Electrostatic Potential (MEP) (Figura 9 (d-f)).

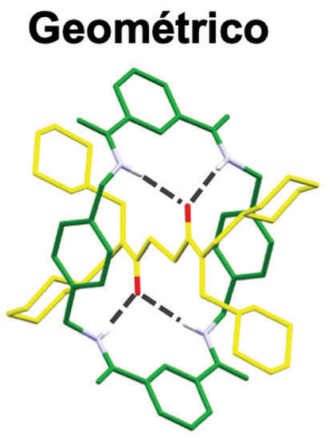

(a)

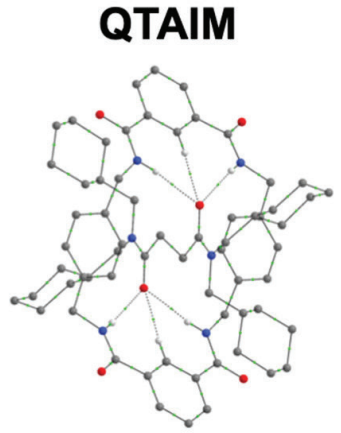

(b)

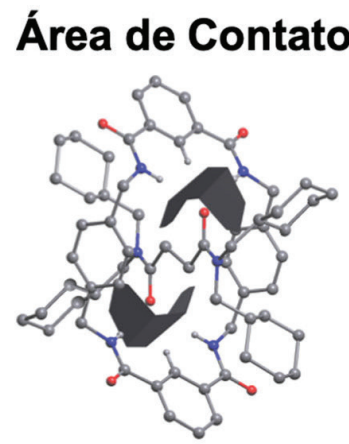

(c)

\section{Superfície de Potencial Eletrostático Molecular}

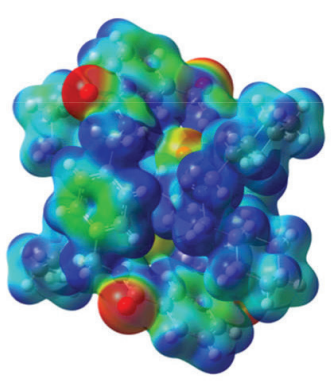

(d)

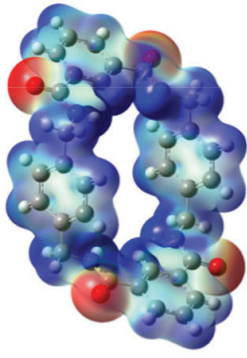

(e)

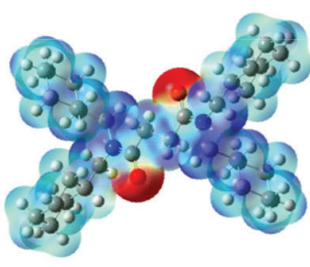

(f)

Figura 9. (a) Parâmetro geométrico (distância átomo-átomo); ${ }^{32}$ (b) caminhos de interação (QTAIM); (c) área de contato do VDP; MEPs do (d) rotaxano e (e) $e(f)$ componentes (vermelho e azul com -0.07 e 0.07 a.u., respectivamente) [reproduzido e adaptado com a permissão de Martins et al., Eur. J. Org. Chem. 2019, 3464-3471. Copyright (2019) Wiley Online Library] 
Nas superfícies de MEPs, os átomos de hidrogênio do macrociclo que participam da ligação de hidrogênio, na cor azul (potencial positivo), interagem com o grupo aceptor no eixo molecular, na cor vermelha (potencial negativo). Esta técnica mostra a complementariedade das superfícies, diferenciando-a das outras técnicas que são mais pontuais. Com a MEP pode-se verificar que existe uma superfície que mutualmente se complementa, não cada interação exercendo seu próprio papel sozinha, mas sim todas as interações cooperando para a estabilização entre as moléculas, ou seja, são "paredes" interagindo e não interações isoladas (Figura 9 (d), (e) e (f)).

De maneira análoga, Orlando e colaboradores ${ }^{38}$ investigaram as diferenças entre polimorfos de [2] rotaxanos em termos de interações intercomponentes. Os autores relataram uma diferença de $8,43 \mathrm{kcal} \mathrm{mol}^{-1}$ em energia de estabilização intercomponente entre as duas formas relatadas, mostrando que uma forma é $14 \%$ mais estável que a outra, quando são correlacionados dados de energia de estabilização. ${ }^{38}$

Adicionalmente, os autores utilizaram da QTAIM para obter mais informações sobre as interações intercomponentes..$^{38}$ Observando que havia uma boa diferença no número total de interações intercomponentes (22 vs. 36) e na contribuição da ligação de hidrogênio trifurcada (41 vs. 60\%) em cada polimorfo. ${ }^{38}$ De maneira geral, foi verificado que no polimorfo que possuía a maior energia de estabilização, a contribuição da ligação trifurcada era menor, pois a energia de estabilização era distribuída em um maior número de interações. Já no polimorfo com menor energia de estabilização, a ligação trifurcada contribuía mais na estabilização, somada a poucas outras interações entre o macrociclo e o eixo molecular. ${ }^{38}$ Em estudos como esse, é possível observar os efeitos das interações intercomponentes na geometria molecular.

\section{CONCLUSÕES E PERSPECTIVAS}

Uma compreensão holística das interações presentes em rotaxanos é fundamental para futuros projetos que possuam essas estruturas como elemento central. Dessa forma, esta revisão demonstrou uma abordagem concisa sobre as interações intercomponentes presentes em moléculas de rotaxanos e como essas interações interveem nas características intrínsecas dessas moléculas, tais como os movimentos por elas executados.

Embora tenhamos passado por avanços nos últimos 50 anos, desde o marco da primeira síntese, o campo de estudo das interações e aplicações dos rotaxanos ainda se encontra no início de seu desenvolvimento. Na constante evolução da área, observa-se um deslocamento do foco de estudo dos trabalhos envolvendo interações intercomponentes. Inicialmente, as interações foram estudadas com o intuito de se obter ou melhorar o modo de interconectar os componentes para a síntese de moléculas de rotaxanos. A partir disso, o foco deslocou-se para o entendimento dos movimentos e aplicabilidade dessas moléculas.

Os estudos em solução demonstram que as interações intercomponentes agem similarmente à um "lubrificante" em uma máquina, facilitando, ou não, o movimento de um componente em relação ao outro. Esse fato é constatado quando, em solução, é observada a influência das interações sobre as barreiras rotacionais, em que um conjunto de interações mais energético faz com que os valores de energia de ativação do movimento aumentem, sendo o contrário verdadeiro.

No estado sólido cristalino, é possível analisar a natureza das interações por diferentes ferramentas, mas observa-se a necessidade de uma visão hermenêutica em relação aos dados obtidos. A complementariedade das superfícies dos componentes do rotaxano e o conjunto de interações envolvidos, devem ser levados em consideração em detrimento de uma visão estreita de se observar apenas interações isoladas e pontuais.
Além disso, procedimentos e metodologias realizadas no Brasil em relação ao avanço dessa área foram destacados. A valiosa experiência acumulada até o momento, deverá resultar na utilização de máquinas moleculares para tarefas úteis em um futuro próximo. Pesquisadores das mais diversas áreas, desde a biologia, química, física, engenharia e ciência dos materiais, estão buscando desenvolver pesquisas com esse caráter. Nesse sentido, abordagens e discussões como as descritas nessa revisão, contribuem para a formação da base de conhecimentos necessários para futuras aplicações dos rotaxanos e no design de novos modelos.

\section{AGRADECIMENTOS}

Os autores agradecem à Fundação de Amparo à Pesquisa do Estado do Rio Grande do Sul (FAPERGS; DOCFIX, 18/2551-0000562-2), ao Conselho Nacional de Desenvolvimento Científico e Tecnológico (CNPq) e a Coordenação de Aperfeiçoamento de Pessoal de Nível Superior (CAPES) pelo apoio financeiro. As bolsas concedidas pelo CNPq (F.F.S.F., M.A.P.M.) e CAPES (T.O., P.R.S.S.; número do processo: 88887.197556/2018-00) também são reconhecidas.

\section{REFERÊNCIAS}

1. Denis, M.; Goldup, S. M.; Nat. Rev. Chem. 2017, 1, 61.

2. Stoddart, J. F.; Angew. Chem., Int. Ed. 2014, 53, 11102.

3. Sluysmans, D.; Stoddart, J. F.; Trends Chem. 2019, 1, 185.

4. Megiatto, J. D.; Guldi, D. M.; Schuster, D. I.; Chem. Soc. Rev. 2020, 49, 8 .

5. Oliveira, N. K.; Salles, T. H. C.; Pedroni, A. C.; Miguita, L.; D’Ávila, M. A.; Marques, M. M.; Deboni, M. C. Z.; Dent. Mater. 2019, 35, 1740.

6. Taveira, S. F.; Varela-Garcia, A.; dos Santos Souza, B.; Marreto, R. N.; Martin-Pastor, M.; Concheiro, A.; Alvarez-Lorenzo, C.; Carbohydr. Polym. 2018, 200, 278.

7. Salles, T. H. C.; Sendyk, D. I.; de Oliveira, N. K.; Machado, D.; Lancellotti, M.; Deboni, M. C. Z.; Kiang, C. T.; d'Ávila, M. A.; Mater. Sci. Eng. C 2017, 77, 912.

8. Ornelas-Megiatto, C.; Becher, T.; Megiatto, J.; Curr. Top. Med. Chem. 2015, 15, 1236.

9. Jover, A.; Budal, R. M.; Meijide, F.; Soto, V. H.; Tato, J. V.; J. Phys. Chem. B 2004, 108, 18850.

10. Stoddart, J. F.; Angew. Chem., Int. Ed. 2017, 56, 11094.

11. Kurniawan, E.; Kom, S.; Kom, M. Em The Nature of the Mechanical Bond; Bruns, C. J., Stoddart, J. F., eds.; John Wiley \& Sons, Inc.: Hoboken, 2016, cap. 1.

12. Neal, E. A.; Goldup, S. M.; Chem. Commun. 2014, 50, 5128.

13. Feu, K. S.; de Assis, F. F.; Nagendra, S.; Pilli, R.; Quim. Nova 2017, 40, 113.

14. Aricó, F.; Chang, T.; Cantrill, S. J.; Khan, S. I.; Stoddart, J. F.; Chem. Eur. J. 2005, 11, 4655.

15. Schalley, C. A.; Weilandt, T.; Brüggemann, J.; Vögtle, F. Em Templates in Chemistry I. Topics in Current Chemistry; Schalley, C. A.; Vögtle, F.; Dötz, K. H., eds.; Springer: Berlin, Heildelberg, 2005.

16. Farrell, A. A.; Kay, E. R.; Bottari, G.; Leigh, D. A.; Jarvis, S. P.; Appl. Surf. Sci. 2007, 253, 6090.

17. Amabilino, D. B.; Stoddart, J. F.; Chem. Rev. 1995, 95, 2725.

18. Martins, M. A. P.; Rodrigues, L. V.; Meyer, A. R.; Frizzo, C. P.; Hörner, M.; Zanatta, N.; Bonacorso, H. G.; Berná, J.; Alajarín, M.; Cryst. Growth Des. 2017, 17, 5845.

19. Hirose, K.; Okada, S.; Umezaki, S.; Young, P. G.; Tobe, Y.; J. Photochem. Photobiol., A 2016, 331, 184.

20. Heim, C.; Affeld, A.; Nieger, M.; Vögtle, F.; Helv. Chim. Acta 1999, 82, 746.

21. Cantrill, S. J.; Fulton, D. A.; Fyfe, M. C. T.; Stoddart, J. F.; White, A. J. P.; Williams, D. J.; Tetrahedron Lett. 1999, 40, 3669. 
22. Anelli, P. L.; Spencer, N.; Stoddart, J. F.; J. Am. Chem. Soc. 1991, 113, 5131 .

23. Fioravanti, G.; Curr. Org. Synth. 2012, 9, 199.

24. Xue, M.; Yang, Y.; Chi, X.; Yan, X.; Huang, F.; Chem. Rev. 2015, 115, 7398.

25. Schill, G.; Zollenkopf, H.; Justus Liebigs Ann. Chem. 1969, 721, 53

26. Vohlídal, J.; Wilks, E. S.; Yerin, A.; Fradet, A.; Hellwich, K.-H.; Hodge, P.; Kahovec, J.; Mormann, W.; Stepto, R. F. T.; Pure Appl. Chem. 2012, $84,2135$.

27. Kay, E. R.; Leigh, D. A.; Zerbetto, F.; Angew. Chem., Int. Ed. 2007, 46, 72.

28. Balzani, V.; Credi, A.; Raymo, F. M.; Stoddart, J. F.; Angew. Chem., Int. Ed. 2000, 39, 3348.

29. Steed, J. W.; Atwood, J. L.; Nano Today 2007, 2, 707.

30. Credi, A.; Ferrer, B.; Pure Appl. Chem. 2005, 77, 1051.

31. Guterres, M. F. A. N.; Ronconi, C. M.; Rev. Virtual Quim. 2009, 1, 10081.

32. Berná, J.; Alajarín, M.; Martínez-Espín, J. S.; Buriol, L.; Martins, M. A. P.; Orenes, R.-Á.; Chem. Commun. 2012, 48, 5677.

33. Orlando, T.; Salbego, P. R. S.; Zimmer, G. C.; Pagliari, A. B.; Bender, C. R.; Rodrigues, L. V.; Bonacorso, H. G.; Zanatta, N.; Berná, J.; Martins, M. A. P.; Eur. J. Org. Chem. 2018, 2018, 4978.

34. Salbego, P. R. S.; Orlando, T.; Farias, F. F. S.; Bonacorso, H. G.; Martins, M. A. P.; Eur. J. Org. Chem. 2019, 2019, 3464.

35. Martinez-Cuezva, A.; Rodrigues, L. V.; Navarro, C.; Carro-Guillen, F.; Buriol, L.; Frizzo, C. P.; Martins, M. A. P.; Alajarin, M.; Berna, J.; J. Org. Chem. 2015, 80, 10049 .

36. Rescifina, A.; Zagni, C.; Iannazzo, D.; Merino, P.; Curr. Org. Chem. 2009, 13, 448 .

37. Cheng, C.; McGonigal, P. R.; Stoddart, J. F.; Astumian, R. D.; ACS Nano 2015, 9, 8672 .

38. Orlando, T.; Salbego, P. R. S.; Taschetto, C. L. R.; Bonacorso, H. G.; Zanatta, N.; Hoerner, M.; Martins, M. A. P.; Cryst. Growth Des. 2019, 19, 1021.

39. Yoon, I.; Benítez, D.; Zhao, Y. L.; Miljanić, O. Š.; Kim, S. Y.; Tkatchouk, E.; Leung, K. C. F.; Khan, S. I.; Goddard, W. A.; Stoddart, J. F.; Chem. - Eur. J. 2009, 15, 1115.

40. Murgu, I.; Baumes, J. M.; Eberhard, J.; Gassensmith, J. J.; Arunkumar, E.; Smith, B. D.; J. Org. Chem. 2011, 76, 688.

41. Jagesar, D. C. C.; Hartl, F.; Buma, W. J. J.; Brouwer, A. M. M.; Chem. - Eur. J. 2008, 14, 1935.

42. Rijs, A. M.; Compagnon, I.; Oomens, J.; Hannam, J. S.; Leigh, D. A.; Buma, W. J.; J. Am. Chem. Soc. 2009, 131, 2428.

43. Alcântara, A. F. P.; Fontana, L. A.; Almeida, M. P.; Rigolin, V. H.; Ribeiro, M. A.; Barros, W. P.; Megiatto, J. D.; Chem. - Eur. J. 2020, 26, 7808.

44. Romero, J. R.; Aragay, G.; Ballester, P.; Chem. Sci. 2016, 8, 491.

45. Cheng, C.; McGonigal, P. R.; Schneebeli, S. T.; Li, H.; Vermeulen, N. A.; Ke, C.; Stoddart, J. F.; Nat. Nanotechnol. 2015, 10, 547.

46. Orlando, T.; Salbego, P. R. S.; Farias, F. F. S.; Weimer, G. H.; Copetti, J. P. P.; Bonacorso, H. G.; Zanatta, N.; Hoerner, M.; Berná, J.; Martins, M. A. P.; Eur. J. Org. Chem. 2019, 2019, 3451.

47. Liu, P.; Shao, X.; Chipot, C.; Cai, W.; Chem. Sci. 2016, 7, 457.

48. Harrison, I. T.; Harrison, S.; J. Am. Chem. Soc. 1967, 89, 5723.

49. Sauvage, J.; Eur. J. Org. Chem. 2019, 2019, 3287.

50. Lim, J. Y. C.; Beer, P. D.; Eur. J. Org. Chem. 2019, 2019, 3433.

51. Brown, A.; Mennie, K. M.; Mason, O.; White, N. G.; Beer, P. D.; Dalton Trans. 2017, 46, 13376.

52. Huang, S.-L.; Hor, T. S. A.; Jin, G.-X.; Coord. Chem. Rev. 2017, 333, 1.

53. Zanichelli, V.; Ragazzon, G.; Orlandini, G.; Venturi, M.; Credi, A.; Silvi, S.; Arduini, A.; Secchi, A.; Org. Biomol. Chem. 2017, 15, 6753.

54. Xu, Y.; Kaur, R.; Wang, B.; Minameyer, M. B.; Gsänger, S.; Meyer, B.; Drewello, T.; Guldi, D. M.; Von Delius, M.; J. Am. Chem. Soc. 2018, 140,13413
55. Chas, M.; Platas-Iglesias, C.; Peinador, C.; Quintela, J. M.; Tetrahedron Lett. 2006, 47, 3119.

56. Toma, S. H.; Toma, H. E.; J. Braz. Chem. Soc. 2007, 18, 279.

57. Chowdhury, S.; Nassar, Y.; Guy, L.; Frath, D.; Chevallier, F.; Dumont, E.; Ramos, A. P.; Demets, G. J. F.; Bucher, C.; Electrochim. Acta 2019, 316,79 .

58. Inthasot, A.; Tung, S. Te; Chiu, S. H.; Acc. Chem. Res. 2018, 51, 1324.

59. Barrell, M. J.; Leigh, D. A.; Lusby, P. J.; Slawin, A. M. Z.; Angew. Chem., Int. Ed. 2008, 47, 8036.

60. Ho, T. H.; Lai, C. C.; Liu, Y. H.; Peng, S. M.; Chiu, S. H.; Chem. - Eur. J. 2014, 20, 4563.

61. Nepogodiev, S. A.; Stoddart, J. F.; Chem. Rev. 1998, 98, 1959.

62. Tepper, R.; Schubert, U. S.; Angew. Chem., Int. Ed. 2018, 57, 6004.

63. Gavina, P.; Tatay, S.; Curr. Org. Synth. 2010, 7, 24.

64. Vögtle, F.; Händel, M.; Meier, S.; Ottens-Hildebrandt, S.; Ott, F.; Schmidt, T.; Liebigs Ann. 1995, 1995, 739.

65. Hirose, K.; Ishibashi, K.; Shiba, Y.; Doi, Y.; Tobe, Y.; Chem. - Eur. J. 2008, 14, 5803.

66. Saura-Sanmartin, A.; Martinez-Espin, J.; Martinez-Cuezva, A.; Alajarin, M.; Berna, J.; Molecules 2017, 22, 1078.

67. Brouwer, A. M.; Frochot, C.; Gatti, F. G.; Leigh, D. A.; Mottier, L.; Paolucci, F.; Roffia, S.; Wurpel, G. W. H.; Science 2001, 291, 2124.

68. Cao, J.; Fyfe, M. C. T.; Stoddart, J. F.; Cousins, G. R. L.; Glink, P. T.; J. Org. Chem. 2000, 65, 1937.

69. Lane, A. S.; Leigh, D. A.; Murphy, A.; J. Am. Chem. Soc. 1997, 119, 11092.

70. Schröder, H. V.; Mekic, A.; Hupatz, H.; Sobottka, S.; Witte, F.; Urner, L. H.; Gaedke, M.; Pagel, K.; Sarkar, B.; Paulus, B.; Schalley, C. A.; Nanoscale 2018, 10, 21425.

71. Bermudez, V.; Capron, N.; Gase, T.; Gatti, F. G.; Kajzar, F.; Leigh, D. A.; Zerbetto, F.; Zhang, S.; Nature 2000, 406, 608.

72. Saura-Sanmartin, A.; Martinez-Cuezva, A.; Pastor, A.; Bautista, D.; Berna, J.; Org. Biomol. Chem. 2018, 16, 6980.

73. Erbas-Cakmak, S.; Leigh, D. A.; McTernan, C. T.; Nussbaumer, A. L.; Chem. Rev. 2015, 115, 10081.

74. Demets, G. J. F.; Quim. Nova 2007, 30, 1313.

75. Wang, S.; Shao, X.; Cai, W.; J. Phys. Chem. C 2017, 121, 25547.

76. Ogoshi, T.; Kotera, D.; Nishida, S.; Kakuta, T.; Yamagishi, T. A.; Brouwer, A. M.; Chem. - Eur. J. 2018, 24, 6325.

77. Kimura, M.; Mizuno, T.; Ueda, M.; Miyagawa, S.; Kawasaki, T.; Tokunaga, Y.; Chem. - Asian J. 2017, 12, 1381.

78. Andersen, S. S.; Share, A. I.; Poulsen, B. L. C.; Kørner, M.; Duedal, T.; Benson, C. R.; Hansen, S. W.; Jeppesen, J. O.; Flood, A. H.; J. Am. Chem. Soc. 2014, 136, 6373

79. Stoddart, J. F.; Acc. Chem. Res. 2001, 34, 410.

80. McGonigal, P. R.; Supramol. Chem. 2018, 30, 782.

81. Leigh, D. A.; Marcos, V.; Nalbantoglu, T.; Vitorica-Yrezabal, I. J.; Yasar, F. T.; Zhu, X.; J. Am. Chem. Soc. 2017, 139, 7104

82. Cheng, C.; McGonigal, P. R.; Liu, W. G.; Li, H.; Vermeulen, N. A.; Ke, C.; Frasconi, M.; Stern, C. L.; Goddard, W. A.; Stoddart, J. F.; J. Am. Chem. Soc. 2014, 136, 14702.

83. Martinez-Cuezva, A.; Carro-Guillen, F.; Pastor, A.; Marin-Luna, M.; Orenes, R.-A. A.; Alajarin, M.; Berna, J.; ChemPhysChem 2016, 17, 1920 .

84. Champin, B.; Mobian, P.; Sauvage, J.-P.; Chem. Soc. Rev. 2007, 36, 358.

85. Nikitin, K.; Lestini, E.; Stolarczyk, J. K.; Müller-Bunz, H.; Fitzmaurice, D.; Chem. - Eur. J. 2008, 14, 1117.

86. Deligkiozi, I.; Papadakis, R.; Tsolomitis, A.; Supramol. Chem. 2012, 24, 333.

87. Altieri, A.; Gatti, F. G.; Kay, E. R.; Leigh, D. A.; Martel, D.; Paolucci, F.; Slawin, A. M. Z.; Wong, J. K. Y.; J. Am. Chem. Soc. 2003, 125, 8644.

88. Fu, N.; Baumes, J. M.; Arunkumar, E.; Noll, B. C.; Smith, B. D.; J. Org. Chem. 2009, 74, 6462 . 
89. Rodrigues, L. V.; Dissertação de Mestrado, Universidade Federal de Santa Maria, Brasil, 2013.

90. Bonacorso, H. G.; Caro, M. S. B.; Zanatta, N.; Martins, M. A. P.; J. Braz. Chem. Soc. 1992, 3, 77.

91. Bonacorso, H. G.; Caro, M. S. B.; Zanatta, N.; Martins, M. A. P.; Quim. Nova 1992, 15, 208.

92. Martins, M. A. P.; Zimmer, G. C.; Rodrigues, L. V.; Orlando, T.; Buriol, L.; Alajarin, M.; Berna, J.; Frizzo, C. P.; Bonacorso, H. G.; Zanatta, N.; New J. Chem. 2017, 41, 13303.

93. Lahlali, H.; Jobe, K.; Watkinson, M.; Goldup, S. M.; Angew. Chem., Int. Ed. 2011, 50, 4151.

94. Sobczyk, L.; Grabowski, S. J.; Krygowski, T. M.; Chem. Rev. 2005, 105, 3513.

95. Baranac-Stojanović, M.; RSC Adv. 2014, 4, 308.

96. Berná, J.; Bottari, G.; Leigh, D. A.; Pérez, E. M.; Pure Appl. Chem. 2007, 79, 39.

97. Anelli, P. L.; Spencer, N.; Stoddart, J. F.; J. Am. Chem. Soc. 1991, 113, 5131.

98. Gholami, G.; Zhu, K.; Baggi, G.; Schott, E.; Zarate, X.; Loeb, S. J.; Chem. Sci. 2017, 8, 7718.

99. Zhu, K.; Vukotic, V. N.; Loeb, S. J.; Angew. Chem., Int. Ed. 2012, 51, 2168.

100. Grabuleda, X.; Jaime, C.; J. Org. Chem. 1998, 63, 9635.

101. Garaudée, S.; Silvi, S.; Venturi, M.; Credi, A.; Flood, A. H.; Stoddart, J. F.; ChemPhysChem 2005, 6, 2145.

102. Du, S.; Fu, H.; Shao, X.; Chipot, C.; Cai, W.; J. Phys. Chem. C 2018 , $122,9229$.

103. Hirva, P.; Haukka, M.; Pakkanen, T. A.; J. Mol. Model. 2008, 14, 879.

104. Alcântara, A. F. P.; Fontana, L. A.; Rigolin, V. H.; Andrade, Y. F. S.; Ribeiro, M. A.; Barros, W. P.; Ornelas, C.; Megiatto, J. D.; Angew. Chem., Int. Ed. 2018, 57, 8979.

105. Li, J.; Sun, J.; Acc. Chem. Res. 2017, 50, 2737.

106. Amini, H.; Baranová, Z.; Weisbach, N.; Gauthier, S.; Bhuvanesh, N.; Reibenspies, J. H.; Gladysz, J. A.; Chem. - Eur. J. 2019, 25, 15896.

107. Li, X.; Yuan, X.; Deng, P.; Chen, L.; Ren, Y.; Wang, C.; Wu, L.; Feng, W.; Gong, B.; Yuan, L.; Chem. Sci. 2017, 8, 2091.
108. Martins, M. A. P.; Frizzo, C. P.; Martins, A. C. L.; Tier, A. Z.; Gindri, I. M.; Meyer, A. R.; Bonacorso, H. G.; Zanatta, N.; RSC Adv. 2014, 4, 44337.

109. Morgon, N. H.; Custodio, R.; Quim. Nova 1995, 18, 44.

110. Berná, J.; Goldup, S. M.; Lee, A. L.; Leigh, D. A.; Symes, M. D.; Teobaldi, G.; Zerbetto, F.; Angew. Chem., Int. Ed. 2008, 47, 4392.

111. Nikolaou, S.; Toma, S. H.; De Souza, V. R.; Alves, J. B.; Araki, K.; Toma, H. E.; Transit. Met. Chem. 2011, 36, 775.

112. Venâncio, M. F.; Nascimento, C. S.; Anconi, C. P. A.; Lopes, J. F.; Rocha, W. R.; Dos Santos, H. F.; De Almeida, W. B.; J. Polym. Sci., Part B: Polym. Phys. 2011, 49, 1101.

113. Anconi, C. P. A.; Nascimento Jr., C. S.; De Almeida, W. B.; Dos Santos, H. F.; J. Braz. Chem. Soc. 2008, 19, 1033.

114. Oliveira, B. G.; Araújo, R. C. M. U.; Ramos, M. N.; Quim. Nova 2010, 33, 1155 .

115. Bader, R. F. W.; Chem. Rev. 1991, 91, 893.

116. Bader, R. F. W.; Atoms in Molecules: A Quantum Theory; Clarendon Press: Oxford, 1994.

117. Pagliari, A. B.; Orlando, T.; Salbego, P. R. S.; Zimmer, G. C.; Hörner, M.; Zanatta, N.; Bonacorso, H. G.; Martins, M. A. P.; ACS Omega 2018, 3, 13850 .

118. Martins, M. A. P.; Meyer, A. R.; Tier, A. Z.; Longhi, K.; Ducati, L. C.; Bonacorso, H. G.; Zanatta, N.; Frizzo, C. P.; CrystEngComm 2015, 17, 7381.

119. Martins, M. A. P.; Hörner, M.; Beck, J.; Tier, A. Z.; Belladona, A. L.; Meyer, A. R.; Zanatta, N.; Bonacorso, G.; Frizzo, C. P.; CrystEngComm 2016, 3866.

120. Copetti, J. P.; Salbego, P. R. S.; Orlando, T.; Rosa, J. M. L.; Fiss, G. F.; de Oliveira, J. P. G.; Vasconcellos, M. L. A.; Zanatta, N.; Bonacorso, H. G.; Martins, M. A. P.; CrystEngComm 2020, 22, 4094.

121. Frizzo, C. P.; Tier, A. Z.; Gindri, I. M.; Meyer, A. R.; Black, G.; Belladona, A. L.; Martins, M. A. P.; CrystEngComm 2015, 17, 4325.

122. Blatov, V. A.; Crystallogr. Rev. 2004, 10, 249.

123. Blatov, V. A.; Shevchenko, A. P.; Proserpio, D. M.; Cryst. Growth Des. 2014, 14, 3576.

124. Guadagnini, P. H.; Bruns, R. E.; Quim. Nova 1996, 19, 148. 